\title{
Le rein immature
}

Le rein fœtal humain est fonctionnel dès la $10^{\text {e }}$ semaine de gestation. Bien que non indispensable à la survie fœtale, il se développe de façon progressive jusqu'à la $35^{\text {e }}$ semaine de gestation, qui marque la fin de la néphrogenèse. Dès la naissance, le rein prend en charge les responsabilités homéostatiques préalablement assurées par le placenta. La naissance est un puissant stimulus du développement de la fonction rénale qui atteindra les valeurs de la maturité vers la fin de la première année de vie. L'intégrité de plusieurs systèmes hormonaux, tels le système rénine-angiotensine et les prostaglandines, est nécessaire au développement rénal, tant dans la vie intraqu'extra-utérine. Les mécanismes responsables de l'homéostasie sont fragiles, et peuvent facilement être perturbés lors de troubles respiratoires et cardiovasculaires, ou lors d'agressions pharmacologiques. La pathogénie et la physiopathologie rénales de ces situations pathologiques commencent à être mieux connues, permettant une approche préventive et thérapeutique plus rationnelle.

\section{ADRESSE}

J.-P. Guignard: professeur de pédiatrie. Centrc hospitalicr universitairc vaudois, service de pédiatrie, CHUV-BH 11, CH-1011 Lausanne, Suisse.

$m / s n^{\circ} 3$ vol. 9, mars 93 e rein a de multiples fonctions : il règle le volume et la composition du liquide extracellulaire, participe au maintien de l'équilibre acido-basique et élimine les déchets du métabolisme azoté. Il active la vitamine $\mathrm{D}$, sécrète l'érythropoiétine et synthétise localement les prostaglandines, l'endothéline, la bradykinine, le $\mathrm{NO}$ et la dopamine. Il est aussi la cible de nombreuses hormones extra-rénales: la vasopressine, l'hormone parathyroïdienne, l'aldostérone, les catécholamines et les glucocorticoïdes. Par l'intermédiaire du bilan sodé et du système rénine- angiotensine, il contribue à la régulation de la tension artérielle. Du point de vue pharmacologique, le rein est la voie d'élimination de nombreux composés actifs ou de leurs métabolites.

Au cours de sa vie intra-utérine, le fœtus n'a guère besoin de ses reins puisque toutes les fonctions homéostatiques sont assurées par le placenta, qui fonctionne comme un véritable rein artificiel. La naissance à terme de nouveau-nés anéphriques confirme cette observation. Bien que non indispensable à la vie fœtale, le rein doit cependant mettre en place ses différentes structures et fonctions 


\section{RÉFÉRENCES}

1. Campbcll S, Wladimiroff JW, Dewhurst CJ. The antenatal measurement of fetal urinc production. $J$ Obstet Gynaecol $\mathrm{Br}$ Commonw 1973; 80 : 680-6.

2. Glick PL, Harrison MR, Golbus MS, et al. Management of the fetus with congenital hydroncphrosis II : pronostic critcria and sclection for treatment. J Pediatr Surg 1985 ; $20: 376-87$

3. Fine RN. Diagnosis and treatment of fetal urinary tract abnormalitics. J Pediatr $1992 ; 121: 333-41$

4. Huttunen NP. Congenital nephrotic syndrome of Finnish typc: a study of 75 paticnts. Arch Iis Child 1976 ; 51 : 344-8.

5. Danpure CJ. Molccular and clinical heterogencity in primary hypcroxaluria typc I. Am J Kidney Dis $1991 ; 17$ : 366-9.

6. Guignard JP, 'lorrado A, Da Cunha O, Gauticr E. Glomerular filtration rate in the first threc wecks of life. J Pediatr $1975 ; 87$ : $268-72$

7. Seikcly M(;, Arant BS Jr. Development of renal hemodynamics : glomerular filtration and renal blood flow. Clin Perinatol $1992 ; 19: 1-13$

8. Guignard JP, Gouyon JB. Adverse cffects of drugs on the immaturc kidncy. Biol Neonate 1988; 53 : 243-52.

9. Heijden VI)AJ, Provoost AP, Nauta J et al. Renal functional impairment in preterm nconates related to intrauterine indomethacinc cxposurc. Pediatr Res 1988 ; 24 : 644-8.

10. Robillard JE. Development of function in fetal kidncy. In : Holliday MA, Barratt 'TM, Vernicr RI, cds. Pediatric Nephro$\log \gamma$. 2nd ed. Baltimore : Williams and Wilkins, 1987: 901-11.

11. Broughton-Pipkin F, Symonds EM, Turner SR. The effect of captopril upon mother and fetus in the chronically cannulated cwe and in the pregnant rabbit. $J$ Physiol 1982; 323: 415-22.

12. Ferris 'TF, Weir EK. Effect of captopril on utcrine blood flow and prostaglandin $\mathrm{E}$ synthesis in the pregnant rabbit. $J$ Clin Invest 1983 ; 71 : 809-15.

13. Plouin PF, Tchobroutsky C. Inhibition de l'enzyme de conversion de l'angiotensine au cours de la grosscssc humainc. Presse Med $1985 ; 14: 2175-8$.

14. Guignard JP. Renal function in the newborn infant. Pediatr Clin North Am 1982 ; 29 : 777-90. de façon à assurer dès la naissance l'homéostasie du nouveau-né. Ce travail décrit quelques-uns des aspects du développement du rein humain et différentes situations cliniques qui peuvent perturber la fonction rénale du nouveau-né.

\section{Développement pendant la vie fotale}

La formation d'urine par le rein fœtal commence avec l'apparition du rein définitif, entre la $9^{e}$ et la $12^{e}$ semaine de gestation. La diurèse, estimée par technique ultrasonographique, est de $10 \mathrm{ml} / \mathrm{h}$ à la $32^{\mathrm{e}}$ semaine de gestation et atteint $28 \mathrm{ml} / \mathrm{h}$ vers le terme [1]. Cette urine est hypotonique, son osmolalité étant voisine de $200 \mathrm{mosm} / \mathrm{kg} \mathrm{H}_{2} \mathrm{O}$. La concentration des électrolytes (sodium, chlore) et de certains solutés (glucose) est bien inférieurc à leur concentration plasmatique, indiquant une réabsorption active de ces solutés au cours du $2^{e}$ trimestre déjà. Cette réabsorption est pertubée lors d'une atteinte rénale secondaire à une uropathie obstructive grave. Chez des foctus humains présentant un obstacle urétral, un mauvais pronostic rénal a été associé à un débit urinaire foctal inférieur à $2 \mathrm{ml} / \mathrm{h}$, une éléva-

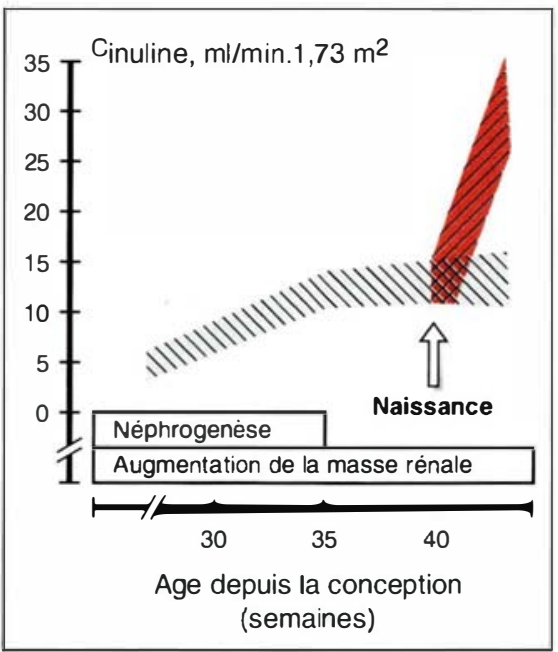

Figure 1. Maturation de la filtration glomérulaire ( $C_{\text {inuline }}$ ) au cours du dernier trimestre de la gestation. Mesure de la clairance de l'inuline chez des nouveau-nés de différents âges gestationnels, étudiés pendant les deux premiers jours de vie. (Adapté d'après [6, 14, 211.) tion des concentrations urinaires de sodium $(>100 \mathrm{mmol} / \mathrm{l})$ et de chlore $(>90 \mathrm{mmol} / \mathrm{l})$ et une augmentation de l'osmolalité urinaire $(>200$ $\left.\mathrm{mosm} / \mathrm{kg} \mathrm{H} \mathrm{H}_{2} \mathrm{O}\right)[2,3]$.

L'urine foctale constitue une partie importante du liquide amniotique. Dans ce liquide peuvent donc se retrouver certains produits signalant une anomalie fœtale. C'est ainsi qu'un diagnostic précoce de syndrome néphrotique congénital [4] peut être suspecté par la présence d' $\alpha$-focto-protéine dans le liquide amniotique. De même, lors d'hyperoxalurie primaire (oxalose), la présence d'acide oxalique dans ce liquide signale un défaut de son métabolisme chez le foctus [5].

La formation de néphrons se développe de façon centrifuge et est, dans l'espèce humaine, achevée vers la $35^{\mathrm{e}}$ semaine de gestation. Le développement de la filtration glomérulaire et de la perfusion rénale suit une évolution caractéristique au cours du dernier trimestre de la grossesse (figure 1). La maturation fonctionnelle est beaucoup plus rapide que la croissance morphologique jusque vers la $35^{\mathrm{c}}$ semaine de gestation. Par la suite, le développement de la filtration glomérulaire se ralentit et se développe de façon parallc̀le à la masse rénale [6].

Plusieurs systèmes hormonaux sont impliqués dans le développement de l'hémodynamique rénale [7]. La survenue d'états oligo-anuriques chez les fœtus de femmes enceintes traitées par l'indométacine ou les inhibiteurs de l'enzyme de conversion [8] est un exemple clinique frappant du rôle joué par les prostaglandines et l'angiotensine II dans le maintien de la fonction rénale du foctus.

\section{Prostaglandines}

L'indométacine, inhibiteur de la synthèse des prostaglandines, est parfois utilisée comme agent tocolytique (pour diminuer les contractions utérines) lors de menace d'accouchement prématuré. Elle traverse rapidement la barrière placentaire, l'équilibre des concentrations maternelles et fotales étant atteint en 5 heures [9]. L'indométacine diminue la diurèse fotale, ce qui, cliniquement, se manifeste par un oligamnios. Une oligo-anurie fatale a parfois été observée chez des 
nouveau-nés de mèrc ayant reçu de l'indométacinc avant la naissance. Bien qu'clle ne soit pas toujours cliniquement évidente, l'atteinte rénale cst probablement très fréquentc. C'cst ainsi que des cstimations précises de la filtration glomérulaire, par mesure de la clairance de l'inuline, ont mis cn évidence unc réduction transitoire de cettc fonction chez 10 nouvcau-nés dont la mère avait reçu de l'indométacine pendant les deux dernicrs jours précédant l'accouchement [9]. Ces observations cliniques montrent l'importance des prostaglandines pour lc développement du rein immature. Des données expérimentales confirment le rôle protecteur des prostaglandines pour le rein foctal. L'indométacinc ct lc méclofénate, un autre inhibiteur de la cyclo-oxygénase, réduisent transitoirement le débit sanguin rénal du foctus de mouton [10]. Ians la même espècc, l'excrétion urinaire des PGE et des PGF2 $\alpha$ s'élève lors d'une hypoxémie foctale, tandis que les inhibiteurs de la synthèse des prostaglandines bloquent la vasodilatation rénale normalement observée dans cettc situation [10].

\section{Angiotensine II}

Les inhibiteurs de l'enzyme de con- version de l'angiotensinc sont des agents hypotenseurs cfficaces. Lcur administration à la femmc enccinte hypertendue a parfois été suivic dc l'apparition d'un oligamnios et de la naissance de nouveau-nés anuriques [8]. Chez ces nouveau-nés, la concentration de l'enzyme de conversion était indétcctable, indiquant que l'inhibiteur de l'cnzyme de conversion (captopril ou énalapril) avait traversé la barrière placentairc. Certains nouvcau-nés anuriques ont survécu grâce à une dialyse péritonéale prolongéc. I a pathogénie de telles anurics a été clarifiée par l'obscrvation des cffets du captopril administré à des brebis ou des lapines gravides. Dans les minutes qui suivent son administration à la brebis, le captopril atteint lc fectus et diminuc fortement sa pression artériclle. Sa demivie chez le foctus est fortement prolongéc par rapport à sa valeur chez la mòre, l'effet hypotenseur persistant pendant 48 heures [11]. Chez la lapine, le captopril diminue significativement la synthèse utérine des prostaglandines et le débit sanguin placentaire, et augmente considérablement la morbidité chez les lapereaux nouveau-nés |12]. Une morbidité élcvée se retrouve en clinique humaine [13]. Ces observations confirment donc l'importance des prostaglandines et de l'angiotensine II pour le maintien de la filtration glomérulaire et de la perfusion rénalc pendant la vic foctalc.

\section{Maturation postnatale}

Filtration glomérulaire et perfusion rénale

Dès la naissance, le rein prend en charge les fonctions homéostatiques assurécs jusqu'alors par le placenta. Lc dévcloppement de la filtration glomérulairc et de la perfusion rénalc, qui s'était ralenti pendant les dernic̀res semaines de gestation, s'accélère à nouveau dès les premic̀res heures postnatales [14]. I a filtration glomérulairc, voisine de $20 \mathrm{ml} / \mathrm{min} \times$ $1,73 \mathrm{~m}^{2}$ chez lc nouveau-né à terme, double pendant les deux premic̀res semaines de vie [6] (figure 2) (chez un adultc dont la surface corporcllc moyenne est de $1,73 \mathrm{~m}^{2}$, la filtration glomérulaire attcint 100 à $120 \mathrm{ml} / \mathrm{min}$ ). I.e développement du flux plasmatique rénal suit une évolution parallèle. Pendant cettc période néonatale, la maturation fonctionnelle est donc à nouveau plus rapide que la croissance morphologique. Partant

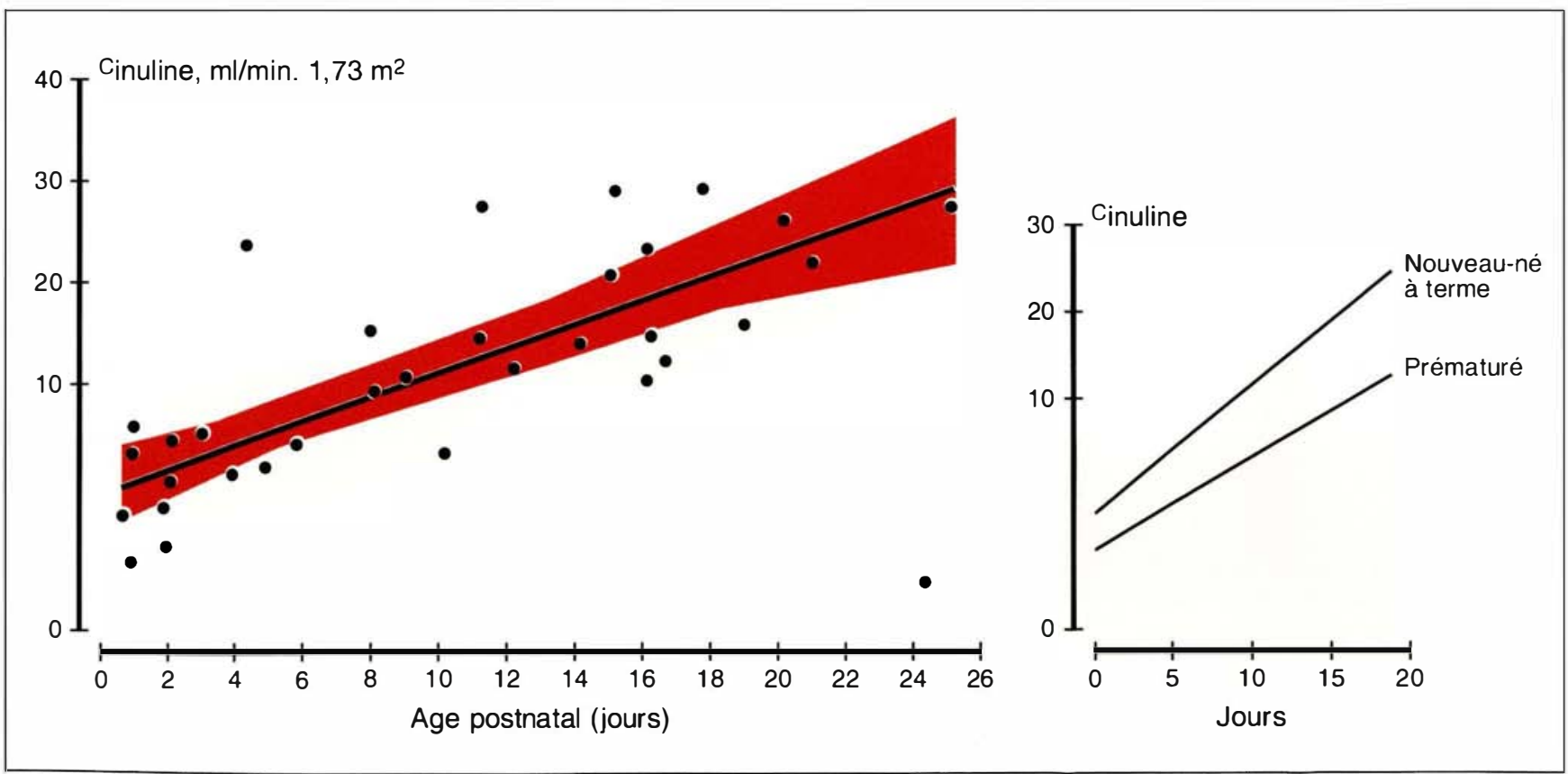

Figure 2. Maturation de la filtration glomérulaire ( $C_{\text {inuline }}$ en fonction de l'âge postnatal. L'évolution de la filtration chez le nouveau-né à terme et chez des prématurés est schématisée dans l'encart. (Adapté d'après [6, 14, 21].) 


\section{RÉFÉRENCES}

15. Gruskin AB, Edclmann CM, Yvan S. Maturational changes in renal blood flow in piglets. Pediatr Res 1976 ; 4 : 7-10.

16. Bailic MD. Development of the endocrinc function of the kidncy. Clin Perinatol 1992 ; 19 : 59-68.

17. Feldman H, Aubert E, Guignard JP Plasma creatinine in the first month of lif c. Arch Dis Child 1982 ; 57 : 123-6.

18. Rudd P'T, Hughes BA, Placzck MM. Reference ranges for plasma creatinine during the first month of life. Arch Dis Child $1983 ; 58: 212-5$

19. Jones DP, Chesncy RW. Development of tubular function. Clin Perinatol $1992 ; 19$ : 33-57.

20. Torrado A, Guignard JP, Prod'hom LS, Gauticr E. Hypoxacmia and renal function in newborns with respiratory distress syndrome (RDS). Helv Paedriatr Acta 1974 ; $29: 3()(9-4() 5$

21. ('uignard JP', John EC. Renal function in the tiny premature infant. Clin Perinatol $1986 ; 13: 377-401$

22. Robillard JE, Segar JL, Smith FG Jose PA. Regulation of sodium metabolism and extracellular fluid volume during development. Clin Perinatol 1992 ; 19 : 15-31.

23. Sulyok E, Varga F, Gyory E, Zetterström R. Postnatal development of renal sodium handling in prematurc infants. $J$ Pediatr 1979 ; 95 : 787-92.

24. Apcria A, Broberger O, Thodenius $\mathrm{K}$ Zettcrström R. Renal responsc to an oral sodium load in newborn full-term infants. Acta Paediatr Scand 1972 ; 61 : 670-6.

25. Schwartz GJ, Haycock GB, Chir B, Edclmann CM, Spitzer A. Late metabolic acidosis : a reasscssment of the definition. J Pediatr $1979 ; 95: 102-7$.

26. Guignard JP, Torrado A, Mazouni SM, Gauticr E. Renal function in respiratory distress syndrome. I Pediatr 1976 ; 88 : 845-50.

27. Broughton-Pipkin F, Smales ORC. A study of factors affecting blood pressure and angiotensin II in newborn infants. I Pediatr $1977 ; 91: 113-9$

28. Robillard JE, Wcismann DN, Ayres NA, Gomez RA, Nakamura KT. Effects of hypoxcmia on renal function during maturation. In : Brodchl J, Ehrich JH, cds. Pediatric Nephrology. Bcrlin : Springer-Verlag, 1984: 51-5. d'un niveau plus bas, la filtration glomérulaire du nouveau-né prématuré se développe de façon presque aussi rapide (figure 2). Cette maturation dépend de modifications anatomiques et hémodynamiques : croissance glomérulaire, élévation de la pression artérielle, diminution de la résistance vasculaire rénale [15], augmentation de la surface de filtration et de la perméabilité capillaire. Elle est aussi associée à des changements importants de la concentration d'hormones vaso-actives : diminution de l'angiotensine II, des prostaglandines, du facteur natriurétique auriculaire et de l'endothéline [16].

Sur le plan clinique, la maturation de la filtration glomérulaire se traduit par des modifications de la créatininémie. A la naissance, le nouveau-né présente des valeurs élevées de créatinine plasmatique qui reflètent la concentration maternelle de ce produit. La créatinine plasmatique du nouveau-né à terme diminue rapidement et se stabilise dès le $5^{\mathrm{e}}$ jour de vic à une valcur voisine dc $35 \mu \mathrm{mol} / \mathrm{l}$ (figure 3) [17]. La filtration glomérulaire du grand prématuré étant beaucoup plus basse, de l'ordrc dc $10 \mathrm{ml} / \mathrm{min} \times 1,73 \mathrm{~m}^{2}$ pour un nouveau-né de 28 semaincs de gestation, le temps nécessaire pour cxcréter l'excès de créatinine maternelle sera plus long, et peut atteindre un mois [18].

\section{Régulations homéostatiques}

Les fonctions tubulaires, pas plus que la filtration glomérulaire, ne sont indispensables à la survie fotale. Leur développement, commencé dès l'apparition du métanéphros, se poursuit au cours des premières semaines postnatales [19].

\section{- Équilibre hydrique}

Le pouvoir de dilution est d'emblée efficace. Le nouveau-né prématuré ou à terme peut, comme l'adulte, diminuer son osmolalité urinaire à des valeurs de $40 \mathrm{mosm} / \mathrm{kg} \mathrm{H}_{2} \mathrm{O}$ [20]. Sa réponse à une surcharge aqueuse est cependant quantitativement limitée, en raison de son faible débit de filtration glomérulaire. La formation d'eau libre nécessite en effet qu'une quantité significative de chlorure de sodium atteigne le site distal de dilution.
Le pouvoir de concentration du nouveau-né est en revanche déficient par rapport aux performances de l'adulte. L'osmolalité urinaire maximale atteinte par le nouveau-né à terme ne s'élève, après déshydratation prolongée $(72 \mathrm{~h})$, qu'à des valeurs d'environ $680 \mathrm{mosm} / \mathrm{kg} \mathrm{H}_{2} \mathrm{O}$, contre 1400 chez l'adulte. Le pouvoir de concentration urinaire est encore plus faible chez le prématuré. Ce défaut de concentration n'est pas dû à un manque de vasopressine endogène. Il a probablement plusieurs causes : (a) le développement incomplet de l'anse de Henlé ; (b) un faible gradient osmotique corticomédullaire, la quantité d'urée disponible pour stockage dans la médullaire rénale étant

\begin{tabular}{l}
\hline GLOSSAIRE * \\
Anurie : tarissement de l'excrétion uri- \\
naire. \\
Filtration glomérulaire : passage \\
d'un ultrafiltrat de plasma sanguin au \\
travers de la paroi des capillaires des \\
glomérules rénaux. L'urine définitive \\
sera obtenue après passage dans les \\
tubules rénaux. \\
Fraction de filltration : la fraction de \\
filtration est le rapport entre le débit de \\
filtration glomérulaire et le flux plasma- \\
tique rénal : FF = DFG/FPR. \\
Inuline : la clairance de l'inuline est \\
une mesure du débit de filtration glo- \\
mérulaire. \\
ADH : hormone antidiurétique. \\
Néphrogenèse : maturation des néph- \\
rons.
\end{tabular}

limitée chez le nouveau-né en fort état d'anabolisme ; (c) une immaturité des systèmes de transport du chlorure de sodium dans l'anse ascendante de Henlé, qui rend aussi compte du faible gradient de concentration corticomédullaire; (d) une immaturité relative du système hormone antidiurétique ( $\mathrm{ADH}$ )adénylate-cyclase qui limite la quantité d'eau libre qui peut être réabsorbée; (e) une interférence des fortes concentrations de prostaglandines endogènes qui, notamment, freinent l'activation de l'AMP cyclique par l'ADH [21]. 
En raison de son faible pouvoir de concentration, le nouveau-né, et plus encore le nouveau-né prématuré, a besoin d'un volume plus important d'eau pour excréter sa charge osmotique quotidienne. Le volume urinaire minimal (et donc l'apport liquidien minimal pour compenser les pertes urinaires) est fonction du pouvoir maximal de concentration urinaire. Si l'osmolalité maximale d'un nouveauné prématuré est de $400 \mathrm{mosm} / \mathrm{kg}$ $\mathrm{H}_{2} \mathrm{O}$, et la charge osmotique à excréter de $15 \mathrm{mosm} / \mathrm{kg} / 24 \mathrm{~h}$, un nouveau-né de $1000 \mathrm{~g}$ aura besoin d'un volume urinaire minimal de $37,5 \mathrm{ml} / 24 \mathrm{~h}$, c'est-à-dire $1,5 \mathrm{ml} / \mathrm{kg} / \mathrm{h}$. $\mathrm{Si}$ la diurèse est inférieure à ce volume, lc nouveau-né entre dans un état d'insuffisance prérénale, avec accumulation de déchets azotés. Lorsque le pouvoir de concentration se développe, le volume urinaire minimal définissant l'oligurie s'élève. Pour excréter une même charge osmotique de $15 \mathrm{mosm} / \mathrm{kg} / 24 \mathrm{~h}$, un nourrisson dont le pouvoir de concentration maximal serait de $850 \mathrm{mosm} / \mathrm{kg} \mathrm{H}_{2} \mathrm{O}$ n'aurait plus besoin que de $17,6 \mathrm{ml} / 24 \mathrm{~h}$, ce qui correspond à une diurèse minimale de $0,7 \mathrm{ml} / \mathrm{kg} / \mathrm{h}$.

\section{- Équilibre sodé}

Le rein joue un rôle primordial dans la régulation de la balance sodéc, et donc dans le maintien de l'osmolalité du volume du liquide extracellulaire ; son excrétion doit être soigneusement contrôlée au niveau rénal [22]. Il existe, chez le nouveau-né comme chez l'adulte, un équilibre remarquable entre la filtration et la réabsorption du sodium. Cet équilibre est réglé par plusieurs systèmes hormonaux : système réninc-angiotensinealdostérone, prostaglandines, facteur natriurétique auriculaire, catécholamines, cortisol [22]. Bien que les mécanismes de régulation de l'excrétion sodée soient présents, leur efficacité n'est pas identique à ce qu'elle est chez l'adulte. Le bilan sodé est souvent négatif, le nouveau-né de petit poids perdant une quantité inappropriée de sel, avec hyponatrémie tardive consécutive. Une diminution de la réabsorption proximale et distale de sodium, malgré les fortes concentrations d'aldostérone [23], est responsable de cet état. Une résistance partielle à l'aldostérone a été suggé- rée. La réponse du nouveau-né à terme face à une surcharge saline cst également moins efficace qu'elle ne l'est chez l'adulte [24]. Chez ce dernier, la réponse natriurétique à la surcharge saline s'effectue essentiellement par une inhibition du transport proximal de sodium. Cette inhibition survient aussi chez le nouveau-né, mais elle est en partie masquée par une réabsorption active de sodium dans le tubule distal, sous l'effet probable de grandes concentrations d'aldostérone circulante. D'autres facteurs, comme le faible débit de filtration glomérulaire, jouent aussi un rôle dans la difficulté qu'a le nouveau-né à éliminer une surcharge sodée.

\section{- Équilibre acido-basique}

La régulation de l'équilibre acidobasique est relativement efficace chez le nouveau-né. Ce dernier excrète, dès les premiers jours de vie, les acides fixes produits par l'oxydation des substrats métaboliques et réabsorbe les bicarbonates filtrés. Il est capable d'abaisser le $\mathrm{pH}$ urinaire lors d'une acidose métabolique [20], les valcurs minimales de $\mathrm{pH}$ étant atteintes dès la $2^{e}$ semainc de vie. En raison d'un seuil d'excrétion urinaire abaissé, le rein du nouveau-né à terme maintient la concentration plasmatique des bicarbonates à des valeurs de

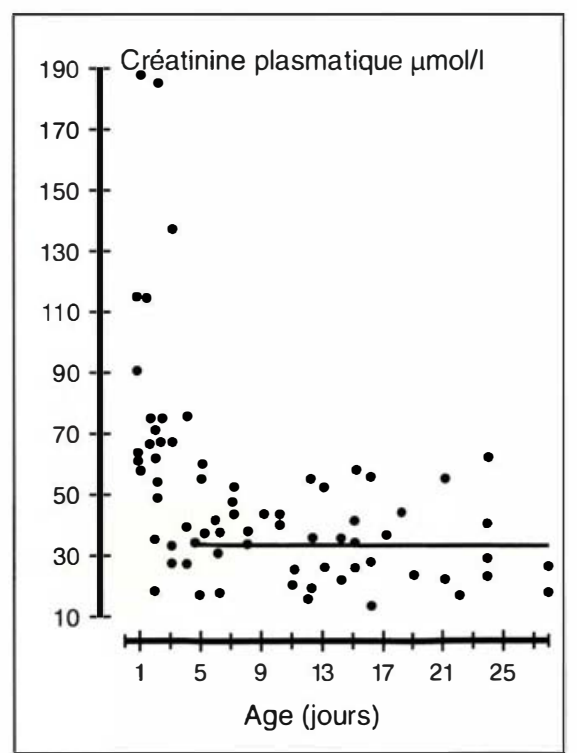

Figure 3. Évolution de la créatinine plasmatique au cours des premières semaines postnatales. (Adapté d'après [17].)
20-22 $\mathrm{mmol} / \mathrm{l}$, et celui du nouveau-né prématuré à des valeurs voisines de $18-20 \mathrm{mmol} / \mathrm{l}$ [25]. Cet abaissement du seuil des bicarbonates ne semble pas dû à un défaut de sécrétion des protons ou à une diminution de l'activité de l'anhydrase carbonique. Il est plus probable que la grande hétérogénéité dans l'état de maturation des néphrons présente à ce stade de vie ou que l'expansion relative du volume extracellulaire soit responsable de l'abaissement du seuil d'excrétion des bicarbonates. Lors d'une surcharge acide exogène, l'excrétion d'ammonium reste inférieure à ce qu'elle est chez l'adulte. Cependant, si le nouveau-né reçoit une alimentation riche en phosphates (lait de vache), le déficit est compensé par une élévation de l'élimination de protons sous forme d'acidité titrable. Le pouvoir d'excrétion acide du prématuré est inférieur à celui du nouveauné à terme. La différence disparaît cependant en trois semaines [14]. Du point de vue pratique, le pouvoir d'acidification du nouveau-né peut donc être considéré comme normal.

Dans l'ensemble, le rein du nouveauné est adapté aux besoins physiologiques. De nombreuses situations endogènes ou iatrogènes, de mĉme que des agressions pharmacologiques, pcuvent toutef ois gravement menacer cette fonction rénale cncorc immature. Nous décrivons ci-dessous quelques-unes de ces situations.

\section{Pathologie rénale néonatale}

Syndrome de détresse respiratoire (SDR)

L'hypoxémie sévère qui est parfois associée au SDR peut avoir des conséquences rénales graves : diminution de la perfusion rénale et de la filtration glomérulaire, dysfonction tubulaire $[20,26]$. L'hypotension, l'hypovolémie, l'acidose respiratoire et l'acidose métabolique qui accompagnent fréquemment le SDR participent certainement à la pathogénie de ces troubles fonctionnels rénaux.

L'un des signes précoces de l'hypoxémie secondaire au SDR est une diminution du débit urinaire qui peut aller jusqu'à l'oligo-anurie [20]. L'osmolalité urinaire s'élève audessus de l'osmolalité plasmatique, 


\section{RÉFÉRENCES}

29. Robillard JE, Wcitmann RE, Burmcister I, Smith FG Jr. Developmental aspects of the renal response to hypoxemia in the lamb fetus. Circ Res 1981; 48 : 128-38.

30). Gouyon JB, Guignard JP. Adenosine in the immature kidncy. Dev Pharmacol Ther $1989 ; 13: 113-9$

31. Guignard JP, Gouyon JB, John EG. Vasoactive factors in the immature kidney. Pediatr Nephrol 1991; 5 : 443-6.

32. Gouyon JB, Guignard JP. 'Theophylline prevents the hypoxemia-induced renal hemodynamic changes in rabbits. Kidney Int $1988 ; 3.3: 1078-8.3$.

33. Gouyon JB, Arnaud M, Guignard JP. Renal effects of low-dose aminophylline and enprofylline in newborn rabbits. Life Sci $1988 ; 42: 1271-8$.

34. Huct F, Grigoras O, Gouyon JB, Guignard JP. Prevention of the hypoxemiainduced renal insufficiency by the angiotensin converting enzyme inhibitor perindoprilat. Kidne'y Int $1992 ; 42$ : 799-80).

35. Kon V, Badr KF. Biological actions and pathophysiologic significance of endothelin in the kidncy. Kidne'y Int 1991 ; 40 11-2.

36. Semama 1), Thonncy M, Gouyon JB Guignard JP. Effects of endothelin on renal function in ncwborn rabbits. Pediatr Res 1993 (sous pressc).

37. Ellison RC, Peckham C.J, I ang P, et al. Evaluation of the preterm infant for patent ductus arteriosus. Pediatrics 1983; 71 : 364-72.

38. Rascher W, Scyberth HW. Atrial natriuretic peptide and patent ductus arteriosus in preterm infants. Arch Dis Child 1987 $62: 1165-7$

39. Heymann MA, Rudolph AM, Silverman NH. Closure of the ductus arteriosus in premature infants by inhibition of prostaglandin synthesis. N Engl J Med 1976 ; 295 : 5.3()$-3$.

40. Guignard JP, Guignard JB, Gouyon JB. Body fluid homeostasis in the newborn inf ant with congestive heart failure : effects of diuretics. Clin Perinatol 1988; 15 : 447-66.

41. Goctzman BV, Sunshinc P, Johnson JD, et al. Neonatal hypoxia and pulmonary vasospasm : response to tolazoline. $J$ Pediatr 1976 ; 89 : 617-21.

42. Naujoks S, Guignard JP. Renal effects of tolazoline in rabbits. Lancet 1979; II : 1075-6. indiquant une perturbation du mécanisme de dilution urinaire. L'oliguric, associéc à unc diminution de la filtration glomérulaire ct de la pcrfusion rénale, est fréqucmment aggravćc par la présence d'une sćcrétion inapproprićc de vasopressinc $[21,26]$. La pcrturbation du mécanismc dc dilution est associéc à d'autres dysfonctions tubulaires : perturbation du mécanismc d'acidification urinaire [20], pertes inappropriécs de sodium et de bicarbonatcs, protéinurie tubulairc significative.

La pathogénie des troubles rénaux induits par l'hypoxémic n'est pas cncore totalement définic. L'autopsie de nouveau-nés décédés de détresse respiratoire ne montre pas de lésions rénales significatives, si ce n'est chcz les nouveau-nćs asphyxićs, qui préscntent parfois une nćcrose corticomćdullaire. L'insuffisancc rénale cst plutôt d'origine prérénalc, différents systèmes hormonaux ct agents vasoconstrictcurs étant libérés par lc strcss hypoxémique. Une stimulation du système rénine-angiotcnsinc a, par cxcmple, ćté démontréc chez elcs nouvcau-nés humains présentant une détressc respiratoire, aussi bicn que chez des animaux nouveau-nćs hypoxiques [27, 28]. Chez le foctus d'agncau, l'hypoxie augmente la libération de catćcholamincs et de vasopressinc [29].

D'autrcs médiateurs de l'insuffisance rénale hypoxémique ont été mis cn ćvidence récemment. C'cst ainsi que l'adénosinc intrarénale [30], qui participe au contrôle de la filtration glomérulairc dans des conditions normales, peut gravement perturber cettc fonction lorsqu'ellc est hyperactivée. Lorsqu'elle agit seule, l'adćnosine cst un vaso-dilatateur de l'artériole postglomérulaire. Elle diminuc donc lc gradient de pression transglomérulairc, avec pour conséquence unc baisse de la filtration glomérulairc [31] et de la fraction de filtration. Quand clle agit cn préscnce de taux ćlevés d'angiotensine II, l'adćnosinc produit aussi une vaso-constriction dc l'artériolc préglomćrulaire. Cc qui a pour conséquence de diminucr davantage encore la filtration glomérulaire, et d'élever la rćsistance vasculaire rénalc. Cc sont bien là les modifications cntraînées par l'hypoxémie.
Ce rôle de l'adénosinc intrarćnale a ćté vérifié dans des expéricnces chez lc lapin nouveau-né ventilé artificicllement de façon à induirc un état d'hypoxémic aiguë. Dans cc modèlc, lc stress hypoxémique diminuc rapidcment la filtration glomérulairc ct la fraction de filtration, ct augmentc la résistancc vasculairc rénalc [32]. L'administration prćalable d'un antagoniste de l'adénosinc, la théophylline, pcrmet de prévenir l'augmentation de la résistancc vasculairc rénale ct la diminution de la filtration glomérulaire [32]. Cette prévention n'cst pas obscrvéc lorsque de l'enprofylline (3-propyl-xanthinc), unc autrc xanthinc qui n'est pas un antagoniste de l'adénosinc, cst utilisćc [33].

L'effet bénćfique de la thćophyllinc confirme l'hypothèse que l'adénosinc intrarćnale cst l'un des médiatcurs de l'insuffisance rénalc hypoxémique. L'angiotensine II participc à la pathogénic de cettc vaso-constriction hypoxémique. En effet, l'administration d'un inhibitcur de l'enzyme de conversion permet, clle aussi, d'atténucr les effets délétères de l'hypoxćmie [34]. L'activation de ces deux agents, adénosinc ct angiotensinc II,

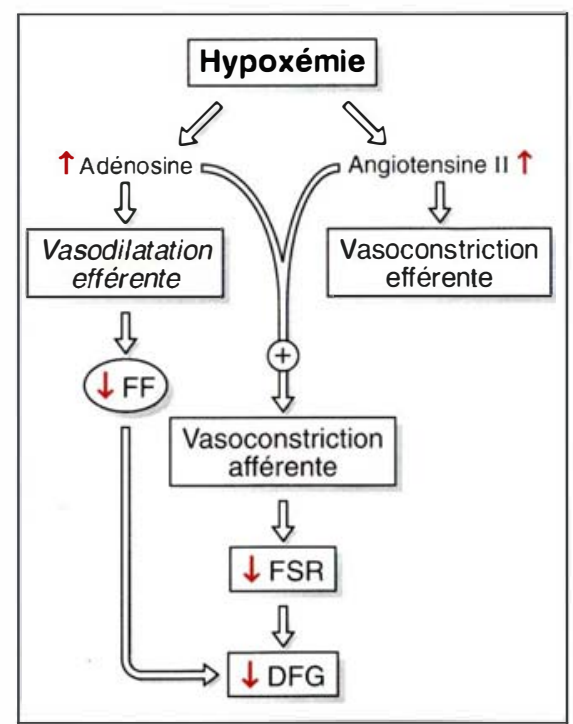

Figure 4. Pathophysiologie rénale de I'hypoxémie. L'activation de deux systèmes vaso-actifs, I'angiotensine II et l'adénosine, est responsable d'une hypoperfusion rénale et d'une diminution de la filtration glomérulaire. FF: fraction de filtration; FSR : flux sanguin rénal; DFG : débit de filtration glomérulaire. 
paraît donc cxpliquer, en grandc partic du moins, les phénomènes hémodynamiques déclenchés par l'hypoxémic (figure 4).

Plus réccmment, unc libération localc d'endothélinc sous l'cffet de l'hypoxic a été misc cn évidence [35]. L'cndothélinc, lc plus puissant vasoconstrictcur connu, paraît donc aussi intervenir dans la vaso-constriction hypoxémique. Unc prévention partielle de ccttc vaso-constriction est obtcnuc par administration d'anticorps anti-cndothéline [36].

La pathogénic de la vaso-constriction hypoxémiquc est complexe. Ellc fait intcrvenir de nombreux agents vasoactifs physiologiques dont l'exaccrbation conduit à une hypoperfusion rénale parfois sévère. L'utilisation d'antagonistes de ces agents permettra ccrtainement de prévenir un jour les conséquences néfastes de l'hypoxémic.

\section{Persistance du canal artériel}

La persistance du canal artéricl s'obscrve chez $20 \%$ des nouveau-nés prématurés [37]. Dans la moitié dc ccs cas, lc shunt gauche-droit cst symptomatique, et produit unc dilatation ventriculairc gauchc, une hypcrtension auriculaire gauche et un œdème pulmonaire. Une hypoxie en résulte ; cn exacerbant la vasoconstriction pulmonaire, elle favorisc à son tour l'apparition d'unc insuffisancc cardiaque droitc [38].

La fcrmeturc du canal artéricl pcut s'obtenir cn inhibant la synthc̀se des prostaglandines [39]. L'indométacine, inhibitcur de la cyclo-oxygénase, est l'agent le plus fréquemment utilisé. Ellc induit parfois des pcrturbations graves de la fonction rénale : diminution de 30 à $40 \%$ de la filtration glomérulairc, diminution plus importante encorc de l'excrétion d'cau libre avcc hyponatrémie consécutive et, de façon plus variable, diminution de l'cxcrétion électrolytique [40]. Ces anomalics fonctionnelles sont transitoires, et durent habituellement dc 2 à 6 jours. L'cxpérimentation animale a confirmé le rôlc important des prostaglandines lors de stress hypoxique rénal. Par lcur pouvoir vasodilatateur de l'artériole afférente, les prostaglandincs améliorent la pcrfusion rénale et la perfusion glomérulaire. Cet cffet bénéfique disparaît $m / s \quad n^{\circ} 3$ vol. 9 , mars 93

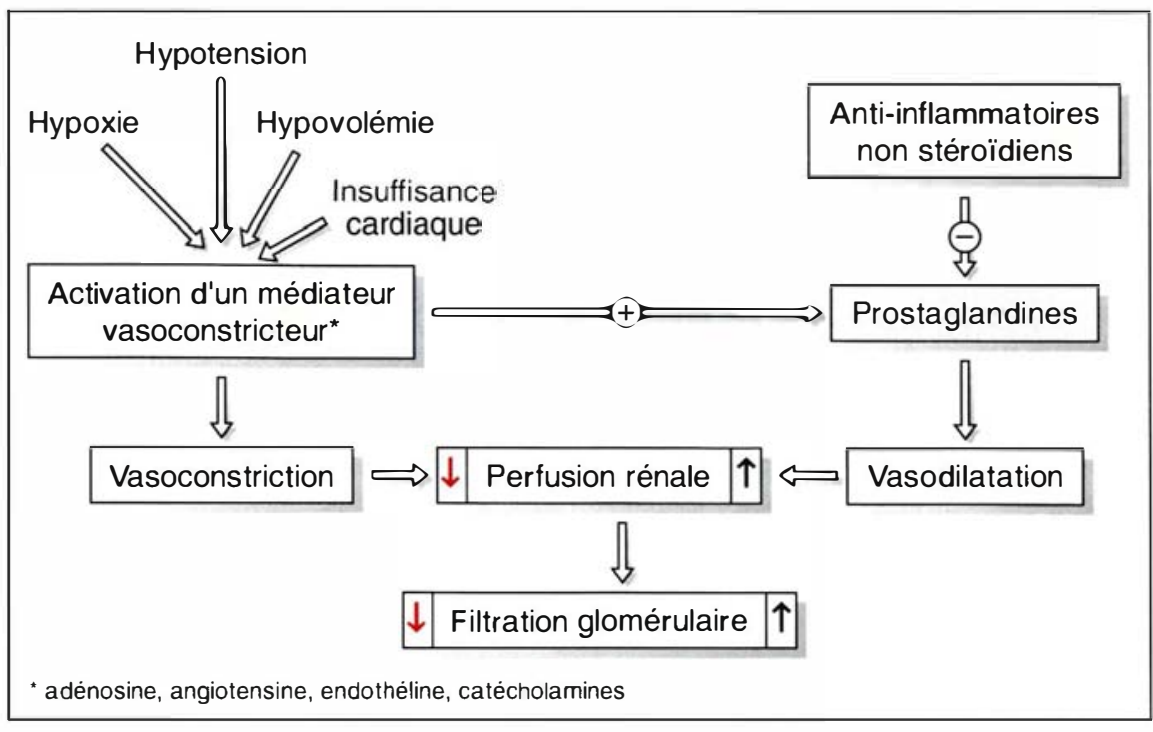

Figure 5. Effet protecteur des prostaglandines lors de stress vasoconstricteur. L'administration d'indométacine inhibe l'effet protecteur des prostaglandines endogènes. (Adapté d'après [31].)

lors d'administration d'indométacinc (figure 5). En raison de son effet délétère sur la filtration glomérulairc, l'indométacine prolonge la demi-vie des médicaments éliminés par filtration, tcls que la digoxine, la vancomycine et les amino-glucosides [40] (Tableau l). Il est donc nécessairc, chcz des patients reccvant de l'indométacinc, d'adapter les dosages de ces médicaments en fonction de la diminution de la filtration glomérulaire, si l'on veut éviter des effets toxiques intempestifs.

Hypertension pulmonaire néonatale La persistance d'une circulation de type fotal s'observe parfois chez le nouveau-né dans les premic̀res heures apròs la naissance. Ellc se manifcste par une cyanosc, une tachypnée et un souffle systolique reflétant un état d'hypcrtension pulmonaire. Elle se caractérise par un shunt gauchcdroit par le foramen ovale et le $d u c$ tus arteriosus, entraînant unc hypoxémie grave. Le chlorhydrate de tolazoline est parfois utilisé dans cette situation, car il entraîne une vasodilatation pulmonaire et améliore les conditions d'oxygénation [41]. Cet $\alpha$ bloquant présente aussi des propriétés histamine-like et un cffct relaxant direct sur le muscle lisse. Il augmente

\begin{tabular}{|l|}
\hline \multicolumn{1}{|c|}{ Tableau I } \\
MÉDICAMENTS ÉLIMINÉS \\
PRINCIPALEMENT \\
PAR VOIE RÉNALE \\
\hline 1. Par filtration glomérulaire \\
- Aminoglucosides \\
gentamicine \\
amikacine \\
tobramycine \\
nétilmycine \\
kanamycine \\
- Vancomycine \\
Digoxine \\
Par sécrétion tubulaire \\
- Acides organiques \\
pénicilline \\
céphalosporines \\
furosémide \\
thiazides \\
Bases organiques \\
procaïnamide \\
triamtérène
\end{tabular}

le débit cardiaque par stimulation sympathicomimétique. Des obscrvations cliniques ont décrit la survenue d'états oligo-anuriques chez des nouveau-nés traités par la tolazolinc. Chcz lc lapin anesthésié, la tolazolinc augmente la résistance vasculaire rénale [42] et diminue fortement la 
perfusion rénale et la filtration glomérulaire. Ce produit, qui conserve des propriétés $\alpha$-agonistes, doit donc être administré avec la plus grande prudence, afin qu'un bénéfice respiratoire ne soit pas obtenu aux dépens d'une vasoconstriction rénale.

\section{Particularités pharmacologiques du rein immature}

La plupart des médicaments ou leurs métabolites sont éliminés par voie rénale. Cette élimination s'effectue par filtration glomérulaire, réabsorption et sécrétion tubulaires (Tableau I, p. 295). La filtration glomérulaire d'un médicament varie selon son degré de liaison aux protéines. Elle diminue lorsque le degré de liaison augmente. La réabsorption des médicaments filtrés s'effectue par diffusion passive ou transport actif. La diffusion passive est le transport le plus répandu. Il dépend du degré d'ionisation du produit, du $\mathrm{pH}$ urinaire et de la diurèse. En plus d'être filtrés, certains médicaments sont sécrétés par les cellules tubulaires. Cette sécrétion résulte d'un transport actif. Deux systèmes sécrétoires distincts transportent les acides et les bases organiques.

Chez le nouveau-né, l'élimination des médicaments excrétés principalement par filtration glomérulaire est relativement inefficace. Ces médicaments comprennent entre autres les aminoglucosides (gentamicine, amikacine, tobramycine, nétilmycine, kanamycine), la vancomycine et la digoxine. Le faible débit de filtration glomérulaire présent chez le nouveau-né rend nécessaire une réduction de la posologie de ces médicaments. Les doses administrées à un grand prématuré doivent être plus faibles encore, ou les intervalles d'administration plus grands. La filtration glomérulaire augmentant rapidement au cours des premières semaines de vie, la posologie des médicaments éliminés par voie rénale doit être adaptée en conséquence [21].

Paradoxalement, la posologie de certains médicaments, comme les thiazides, doit être élevée en raison du faible débit de la filtration glomérulaire. Ces agents ont en effet besoin, suffisante du médicament atteigne le site distal du néphron où s'exerce leur action.

Les médicaments éliminés par sécrétion tubulaire comprennent les acides organiques (pénicilline, céphalosporine, furosémide, thiazides) et les bases organiques (procaïnamide, triamtérène). En raison de l'immaturité des processus de sécrétion tubulaire, ces médicaments ont également une demi-vie prolongée chez le nouveau-né. La pénicilline en est un exemple. L'élimination rénale de la pénicilline peut être stimulée par le substrat. Lors d'administration chronique, sa clairance peut doubler, nécessitant une adaptation des doses. Le phénomène de stimulation de son propre transport par la pénicilline ne semble intervenir qu'au stade d'immaturité tubulaire.

Une élimination ralentie est aussi probablement responsable de la longue durée d'action de nombreux médicaments tels que l'indométacine, l'acide salicylique, le paracétamol ou les sulf amides. Le dosage de tous ces médicaments doit par conséquent être diminué chez le nouveau-né si l'on veut éviter des effets toxiques. Chcz le nouveau-né à risque, de nombreux médicaments vaso-actifs peuvent altérer la filtration glomérulaire : indométacine, tolazoline, inhibiteurs de l'enzyme de conversion, produits de contraste radiologique [8]. Une adaptation de la posologie des autres médicaments administrés simultanément est donc nécessaire. Lorsqu'elle est possible, la détermination de la concentration des médicaments doit être obtenue.

De façon surprenante, les aminoglucosides, fortement néphrotoxiques chez l'adulte, semblent relativement bien tolérés par le nouveau-né prématuré. Il est possible que l'immaturité des néphrons superficiels, en particulier la croissance inachevée de certains segments tubulaires chez le grand prématuré, protège ce dernier contre les effets toxiques des aminoglucosides. En effet, la toxicité de ces agents passe par une accumulation au niveau du parenchyme rénal. Bien que relativement inoffensifs en apparence, les aminoglucosides devraient cependant être utilisés avec prudence. Il est en effet possible que leur apparente innocuité soit, en partie, due à l'imprécision des méthodes de détermination de la filtration glomérulaire utilisées chez le nouveau-né

\section{Summary}

The immature kidney

In humans, urine formation starts with the metanephros at the 10 th week of gestation. Nephrogenesis progresses during gestation and is achieved around the 35th week. Clamping of the cord is the signal for a striking increase in renal function which reaches mature levels at the end of the first year of life. The integrity of several hormonal systems (the reninangiotensin system, the prostaglandins) is mandatory for kidney growth and the development of renal function. The mechanisms underlying renal homeostasis are fragile and can easily be disturbed during respiratory and cardiovascular distress, or be affected by the administration of vasoactive agents. Thus, perinatal asphyxia or hypoxemia, as seen in respiratory distress syndrome or neonatal pulmonary hypertension induces intense renal vasoconstriction, with consequent oligoanuria. Congestive heart failure also results in renal hypoperfusion and sodium retention. Vasoactive agents and diuretics (indomethacin, tolazoline, furosemide) used to treat these conditions can result in renal vasoconstriction, renal hypoperfusion and failure. The pathogenesis and pathophysiology of neonatal renal disturbances being now better defined, a rational approach to the treatment of renal functional abnormalities during the neonatal period is possible.

\section{TIRÉS A PART}

J.-P. Guignard. 